\title{
Posterior Mediastinal Chondrosarcoma. A Rare Entity
}

\section{Abstract}

Introduction: Chondrosarcoma is a well defined tumor of soft tissue with calcification. Extraskeletal myxoid chondrosarcoma is an unusual sarcoma of soft tissue adding only 3\% to all soft tissue tumors. Majority of mediastinal foci originates from variety of tissues, the reason being their diverse embryological and anatomical approximations. Chondrosarcomas are more common in males with 2: 1 male to female ratio. The exact pathology of these tumors is unclear; however recent data ensures that these tumors have multidirectional delineation. Classical histopathological features of chondrosarcoma include S-100 positivity, EMA positivity. These features of Immunohistochemical favor extra skeletal myxoid chondrosarcoma.

Case Report: Recent data signifies that the tumor is known for its rare occurrence, here we have reported a unique case of 40 years old male visited Abbasi Shaheed Hospital for pre employment checkup without any symptoms. All the tests were negative except Chest $X$ ray PA view which revealed dense mass on lower lobe of lung. For further evaluation, CT scan of chest with contrast was ordered and eventually the mass was resected surgically. On the basis of macro and microscopic findings, histopathological tests and immunohistochemical stains, the mass was found to be chondrosarcoma with myxoid origin. This tumor has to be distinguishing among the list of different diseases like hamartoma, hydatid cyst, and neuroendocrine tumors for the differential diagnosis of the case.

Conclusion: Our paper reports an extraskeletal mesenchymal chondrosarcoma originating in the posterior mediastinum with a rare presentation. 


\section{Introduction}

Ewing's sarcoma, osteosarcoma and chondrosarcoma constitute the most common primary bone tumors of pelvis [1, 2]. Chondrosarcomas constitutes a speckled group of neoplasias that produces cartilage matrix aberrantly [3]. Major fractions of these tumors grow slowly and rarely metastasize, and have an excellent post operative prognosis. The reason of this might be the lack of adequate vascular supply, low mitotic division rate, and extra cellular matrix; which devise them relatively less sensitive to chemotherapy and radiotherapy. Surgical expurgation is the most recommended treatment for medium- to high-grade tumors. However, in chondrosarcomas with metastatic potential, surgery is not indicated and thus only palliative treatment is recommended. Other restrictions for surgical excision for chondrosarcomas include origin of tumor from trunk or approximation of tumor near vital anatomic structures. The various subtypes of chondrosarcomas are rare and however the known varieties are categorized as neuronal (nerve) and muscular. A small group of patients often presents with metastatic disease. In up to $13 \%$ of recurring chondrosarcomas, metastatic foci are at higher grade than the earliest occurring neoplasm $[4,5,6,7,8]$. The extent and mode of calcification of the matrix are variable. They may range from just focus of rings and arcs to diffuse and granular calcified masses accompanied by considerable elements of soft tissue, bone invasion and destruction $[6,7,8]$. The presentation of the disease is variable ranging from asymptomatic to an anterior mediastinal mass, leading to non conforming clinical symptoms. Most common age of presentation is middle age in both genders except for rhabdoymosarcoma that presents more frequently in pediatric age group. The most common tumor presentation is non specific pain experienced by $95 \%$ of patients; a sensation of fullness has also been regarded in $30-80 \%$ of patients [9]. Secondary tumors are more common than primary mediastinal tumors. These include tumors of vertebral body, or components of chest wall and ribs. Aoki et al [10] reviewed 250 histopathologically established cases of chondrosarcoma, in which he was able to just localize $4 \%$ originating from sternum alone. However, primary tumors originating from sternum are far less common than sternal metastases having origin from lungs, thyroid and renal carcinomas [11].

\section{Case presentation}

A 40-year-old man without any history of symptoms was diagnosed with a S100- and EMA positive myxoid Chondrosarcoma. He visited Abbasi Shaheed Hospital for pre employment checkup. All the baseline investigations were conducted which were all negative as expected, except Chest X-ray. Evaluation of Chest $X$-ray PA view illustrated a dense mass on lower lobe of left lung. His past history was insignificant. Initially the soft tissue density mass lesion was examined in the retro cardiac area on the PA chest $X$ - ray which appears to be posterior in left lower lobe. The mass appeared to be well defined and with benign gaze. However CT correlation was strongly advised. Later in the CT chest with contrast, the mass appeared to be a large well defined rounded encapsulated low attenuation lesion with associated soft tissue competent, calcification and internal septations in the left para vertebral location lying in the posterior mediastinal flat measuring approximately $59 \times 51 \mathrm{~mm}$. Medially it was adjacent to T9 vertebral body and its adjacent nerve. However no evidence of neural foraminal extension or neural canal widening was found. It was also closely abutting the aorta and the esophagus. Laterally it was lying near left lower lobe with minimal adjacent atelectatic changes. The findings are most likely representing a neurogenic tumor. However other less likely differentials would include hydatid cyst or hamartoma. He underwent segmental resection of mass resulting in complete removal of gross disease which was then confirmed by CT scan. 
In the later gross picture of histopathology, the specimen in formalin coded appeared as an oval and brown mass measuring $7.5 \times 7.3 \mathrm{~cm}$ weighing $68 \mathrm{gm}$. The cut surface showed solid areas with hemorrhages, necrosis and central areas of cystic degeneration. Microscopically a well circumscribed neoplastic lesion with thick fibrous capsule was found. The cells were arranged in strands, cords, and gland like structures with a myxoidy background. The cells were oval having pleomorphic nuclei with inconspicuous to variably prominent nucleoli and moderate eosinophilic cytoplasm. The tumor was $0.1 \mathrm{~cm}$ away from the painted capsule. Immunohistochemical stains show S-100 positive, EMA positive, Cytokeratin CAM 5.2 negative, Desmin negative, ASMA negative reactivity patterns. These Immunohistochemical tests favored diagnosis as extra skeletal myxoid chondrosarcoma. The list of differential diagnosis includes cellular myxoma, reactive myxoid lesions, myoepithelioma, myxoid neurofibroma and neurothekeoma. An exclusive case of primary myxoid chondrosarcoma in posterior mediastinum was reported by Pescarmona [11]. The exclusivity of the tumor was due to it dumbbell appearance [11]. In a case presented by Sang Seok, immunohistochemical features presented by extra mesenchymal chondrosarcoma included S100 protein positive, Vimentin positive and CD 99 positive [12]. Suster $S$ reported 6 cases of primary malignant mesenchymal tumors of posterior mediastinum presenting with soft tissue masses. The lesions ranged from spectrum of diseases including mesenchymal chondrosarcoma to EMC. One of these six patients with myxoid chondrosarcoma developed bilateral pulmonary metastasis 10 months post operatively. Such findings reveal significant potentials for soft tissue tumors to develop propensity for distant metastasis [12].

\section{Conclusion}

Immunohistochemical stains showing S-100 positive, EMA positive, Cytokeratin CAM 5.2 negative, Desmin negative, ASMA negative reactivity patterns are features of extra skeletal myxoid chondrosarcoma.

\section{Consent}

Written informed consent was obtained from the patient for publication of this Case report and any accompanying images. A copy of the written consent is available for review by the Editor-in-Chief of this journal.

\section{Conflicts of interests}

'The author(s) declare that they have no competing interests'.

\section{Abbreviation \\ Extra mesenchymal chondrosarcoma}




\section{References}

1. Shin KH, Rougraff BT, Simon MA. Review Oncologic outcomes of primary bone sarcomas of the pelvis. Clin Orthop Relat Res. 1994 Jul; (304): 207-17

2. Kawai A, Healey JH, Boland PJ, Lin PP, Huvos AG, Meyers PA. Prognostic factors for patients with sarcomas of the pelvic bones. Cancer. 1998 Mar 1; 82(5): 851-9

3. Fletcher CDM, Unni KK, Mertens F, editors.World Health Organization Classification of Tumor. Pathology and Genetics. Tumor of Soft Tissue and Bone. Lyon, France: IARC Press; 2002. Cartilage tumor; p. 234-257.

4. Bjornsson J, McLeod RA, Unni KK, et al Primary chondrosarcoma of long bones and limb girdles. Cancer 1998; 83: 2105-2119.

5. Hideo M, Hirro Y, Shinjiro K, et al. Large chondrosarcoma of the rib invading the mediastinum and the spine. J Thorac Cardiovasc Surg. 2006 Oct; 132(4): 986-7.

6- Angulo Hervías E, Lample Lacasa C, Crespo Rodríguez AM, Yagüe Romeo D. Primary mesenchymal chondrosarcoma of the mediastinum. Radiologia. 2007 Sep-Oct; 49(5): 358-61.

7. Chetty R. Primary chondrosarcoma of the lung. A clinicopathologic study. Cancer. 1993 Jul 1; 72(1): 69-74.

8. Hayashi T, Tsuda N, Iseki M, Kishikawa M, Shinozaki T, Hasumoto M. Primary chondrosarcoma of the lung. A clinicopathologic study. Cancer. 1993 Jul 1; 72(1): 69-74.

9. Nasseri F, Chen GJ, Nachiappan AC. Case 195: chondrosarcoma of the posterior mediastinum. Radiology. 2013 Jul; 268(1): 299303.

10. Aoki J, Moser RP, Kransdorf MJ. Chondrosarcoma of the sternum: CT features. J Comput Assist Tomogr. 1989; 135: 806810.

11. Ashford RU, Stanton J, Khan F, et al. Surgical treatment of chondrosarcoma of the sternum. Sarcoma. 2001; 54: 209-213.

12. Sang Seok Jeong, Phil Jo Choi, Dong Won Kim, et al. Primary Extraskeletal Mesenchymal Chondrosarcoma of the Anterior Mediastinum. Korean J Pathol. 2013 Oct; 47(5): 492-494.

13. Suster S, Moran CA. Malignant cartilaginous tumors of the mediastinum: clinicopathological study of six cases presenting as extraskeletal soft tissue masses. Hum Pathol. 1997 May; 28(5): 588-94

\section{Comment on this article:}

\section{(f) $[$ in $8+\boldsymbol{S} P$}

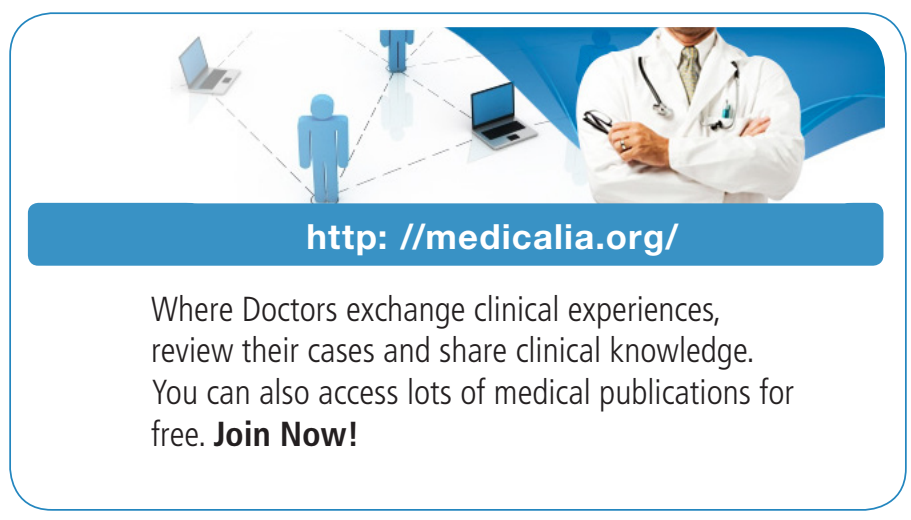

\section{Publish with iMedPub}

http: //www.imed.pub

International Archives of Medicine is an open access journal publishing articles encompassing all aspects of medical science and clinical practice. IAM is considered a megajournal with independent sections on all areas of medicine. IAM is a really international journal with authors and board members from all around the world. The journal is widely indexed and classified Q1 in category Medicine. 